Pacific Journal of Mathematics

FRACTIONAL ELEMENTS IN MULTIPLICATIVE LATTICES 


\title{
FRACTIONAL ELEMENTS IN MULTIPLICATIVE LATTICES
}

\author{
RICHARD G. BURTON
}

An abstract study of the theory of fractional ideals of a commutative ring is begun. In particular, the definition of principal element in a multiplicative lattice $L$ is used to define a lattice of fractional elements, $L^{*}$, associated with $L$. As one application of this definition a theory of Dedekind lattices is developed. This construction also allows the development of an abstract theory of integral closure for a Noether lattice. This theory will be presented in a further paper.

By a multiplicative lattice we mean a complete lattice $L$ together with a commutative, associative multiplication on $L$ such that (i) $a(b \cup c)=a b \cup a c$ and (ii) $a b \leqq a \cap b$ for all $a, b, c$ in $L$. We further assume that $L$ has a greatest element $e$ such that $e a=a$ for all $a$ in $L$ and a least element 0 . We denote the meet and join of two elements $a, b$ in $L$ by $a \cup b$ and $a \cap b$, respectively, and we use $\leqq$ to denote the order relation on $L$. A lattice with a multiplication satisfying condition (i) above is a lattice ordered semi-group.

An element $m$ in $L$ is join principal if $(a \cup b m): m=a: m \cup b$ for all $a, b$ in $L$, meet principal if $(a \cap b: m) m=a m \cap b$ for all $a, b$ in $L$, and principal if it is both join and meet principal. This definition of principal element was given by Dilworth in [1].

The author wishes to express appreciation to Dr. William M. Cunnea.

1. Definition and basic properties of $L^{*}$. Let $L$ be a multiplicative lattice and consider the set of all ordered pairs of the form $(p, q)$, where $p, q \in L$ and $q$ is a principal nonzero divisor of $L$. We define a relation, denoted by " $\sim$ ", on this set as follows:

$$
(p, q) \sim\left(p^{\prime}, q^{\prime}\right) \quad \text { iff } \quad p q^{\prime}=q p^{\prime} .
$$

LEMMA 1.1. “ " is an equivalence relation on the set of ordered pairs defined above.

Proof. It is clear that the relation is reflexive and symmetric. To show transitivity, assume $(p, q) \sim\left(p^{\prime}, q^{\prime}\right)$ and $\left(p^{\prime}, q^{\prime}\right) \sim\left(p^{\prime \prime}, q^{\prime \prime}\right)$. Then $p q^{\prime} q^{\prime \prime}=q p^{\prime} q^{\prime \prime}$ and since $p^{\prime} q^{\prime \prime}=q^{\prime} p^{\prime \prime}$ this can be rewritten $p q^{\prime \prime} q^{\prime}=$ $q p^{\prime \prime} q$. Therefore, 


$$
p q^{\prime \prime}=p q^{\prime \prime} q^{\prime}: q^{\prime}=q p^{\prime \prime} q^{\prime}: q^{\prime}=q p^{\prime \prime},
$$

where the first and last equalities follow from the fact that $q^{\prime}$ is a principal nonzero divisor in $L$.

Let $L^{*}$ denote the set of equivalence classes defined by the above equivalence relation. We denote the equivalence class containing $(p, q)$ by $\langle p, q\rangle$. If $\langle p, q\rangle$ and $\langle r, s\rangle$ are elements of $L^{*}$ we define $\langle p, q\rangle \leqq\langle r, s\rangle$ iff $p s \leqq q r$.

Lemma 1.2. The relation " $\leqq$ is a partial order on $L^{*}$.

Proof. To show that " $\leqq$ " is well defined, assume that $(p, q) \sim$ $\left(p^{\prime}, q^{\prime}\right)$ and $(r, s) \sim\left(r^{\prime}, s^{\prime}\right)$. Then $p q^{\prime}=q p^{\prime}$ and $r s^{\prime}=s r^{\prime}$. Now, suppose $p s \leqq q r$. Then

$$
\left(p^{\prime} s^{\prime}\right) q s=s^{\prime} q^{\prime} p s \leqq s^{\prime} q^{\prime} q r=\left(r^{\prime} q^{\prime}\right) q s
$$

Therefore, since $q s$ is a principal nonzero divisor in $L$,

$$
p^{\prime} s^{\prime}=\left[\left(p^{\prime} s^{\prime}\right)(q s)\right]:(q s) \leqq\left[\left(r^{\prime} q^{\prime}\right)(q s)\right]:(q s)=r^{\prime} q^{\prime}
$$

and " $\leqq$ " is well defined.

It is clear the relation is reflexive and antisymmetric. To show transitivity, suppose $\langle p, q\rangle \leqq\langle r, s\rangle$ and $\langle r, s\rangle \leqq\left\langle r^{\prime}, s^{\prime}\right\rangle$. Then $p s s^{\prime} \leqq$ $q r s^{\prime} \leqq q s r^{\prime}$. Thus,

$$
p s^{\prime}=p s^{\prime} s: s \leqq q r^{\prime} s: s=q r^{\prime} .
$$

THEOREM 1.1. The set $L^{*}$ together with the relation $\leqq$ is a lattice with least upper bound and greatest lower bound given by the following equations :

(1) $\langle p, q\rangle \cup\left\langle p^{\prime}, q^{\prime}\right\rangle=\left\langle p q^{\prime} \cup q p^{\prime}, q q^{\prime}\right\rangle$

(2) $\langle p, q\rangle \cap\left\langle p^{\prime}, q^{\prime}\right\rangle=\left\langle p q^{\prime} \cap q p^{\prime}, q q^{\prime}\right\rangle$.

Proof. Let $\langle p, q\rangle$ and $\left\langle p^{\prime}, q^{\prime}\right\rangle$ be any two elements of $L^{*}$. Then

$$
p q q^{\prime} \leqq p q q^{\prime} \cup q q p^{\prime}=q\left(p q^{\prime} \cup q p^{\prime}\right)
$$

Therefore,

$$
\langle p, q\rangle \leqq\left\langle p q^{\prime} \cup q p^{\prime}, q q^{\prime}\right\rangle . \quad \text { Similarly, }
$$$$
\left\langle p^{\prime}, q^{\prime}\right\rangle \leqq
$$
$\left\langle p q^{\prime} \cup q p^{\prime}, q q^{\prime}\right\rangle$.

Thus, $\left\langle p q^{\prime} \cup q p^{\prime}, q q^{\prime}\right\rangle$ is an upper bound for $\langle p, q\rangle$ and $\left\langle p^{\prime}, q^{\prime}\right\rangle$. Moreover, if $\langle p, q\rangle \leqq\langle r, s\rangle$ and $\left\langle p^{\prime}, q^{\prime}\right\rangle \leqq\langle r, s\rangle$, then $p s \leqq q r$ and $p^{\prime} s \leqq q^{\prime} r$. Therefore 


$$
\left(p q^{\prime} \cup q p^{\prime}\right) s=p q^{\prime} s \cup q p^{\prime} s \leqq q q^{\prime} r \cup q q^{\prime} r=q q^{\prime} r .
$$

Thus, $\left\langle p q^{\prime} \cup q p^{\prime}, q q^{\prime}\right\rangle \leqq\langle r, s\rangle$ and $\left\langle p q^{\prime} \cup q p^{\prime}, q q^{\prime}\right\rangle$ is the least upper bound for $\langle p, q\rangle$ and $\left\langle p^{\prime}, q^{\prime}\right\rangle$.

Since $q$ is a principal nonzero divisor,

$$
\left(p q^{\prime} \cap q p^{\prime}\right) q=\left(p q^{\prime} \cap\left(q p^{\prime} q\right): q\right) q=p q^{\prime} q \cap q p^{\prime} q \leqq q q^{\prime} p
$$

Thus, $\left\langle p q^{\prime} \cap q p^{\prime}, q q^{\prime}\right\rangle \leqq\langle p, q\rangle$ and a similar argument shows that $\left\langle p q^{\prime} \cap\right.$ $\left.q p^{\prime}, q q^{\prime}\right\rangle \leqq\left\langle p^{\prime}, q^{\prime}\right\rangle$.

If $\langle r, s\rangle \leqq\langle p, q\rangle$ and $\langle r, s\rangle \leqq\left\langle p^{\prime}, q^{\prime}\right\rangle$, then $r q \leqq s p$ and $r q^{\prime} \leqq$ $s p^{\prime}$. Therefore, since $s$ is a principal nonzero divisor,

$$
s\left(p q^{\prime} \cap q p^{\prime}\right)=s p q^{\prime} \cap s q p^{\prime} \leqq r q q^{\prime} \cap r q q^{\prime}=r q q^{\prime} .
$$

Thus, $\left\langle p q^{\prime} \cap q p^{\prime}, q q^{\prime}\right\rangle$ is the greatest lower bound of $\langle p, q\rangle$ and $\left\langle p^{\prime}, q^{\prime}\right\rangle$.

Definition 1.1. The lattice $L^{*}$ will be called the lattice of fractional elements of $L$.

We now define a multiplication on $L^{*}$ as follows: If $\langle p, q\rangle$ and $\langle r, s\rangle$ are elements of $L^{*}$, then

$$
\langle p, q\rangle\langle r, s\rangle=\langle p r, q s\rangle
$$

It is easy to see that this multiplication is well defined.

Proposition 1.1. With the above multiplication, $L^{*}$ is a commutative, associative lattice ordered semigroup. The element $\langle e, e\rangle$ is a multiplicative identity. have

Proof. For arbitrary elements $\langle a, b\rangle,\langle c, d\rangle$, and $\langle f, g\rangle$ in $L^{*}$ we

$$
\begin{aligned}
\langle a, b\rangle(\langle c, d\rangle \cup\langle f, g\rangle) & =\langle a, b\rangle\langle c g \cup d f, d g\rangle=\langle a c g \cup a d f, b d g\rangle \\
& =\langle b(a c g \cup a d f), b(b d g)\rangle=\langle a c, b d\rangle \cup\langle a f, b g\rangle \\
& =\langle a, b\rangle\langle c, d\rangle \cup\langle a, b\rangle\langle f, g\rangle,
\end{aligned}
$$

where we have used the fact that

$$
(b(a c g \cup a d f), b(b d g)) \sim(a c g \cup a d f, b d g) .
$$

Commutativity and associativity for multiplication are obvious as is 
the fact that $\langle e, e\rangle$ is a multiplicative identity.

We remark that $L^{*}$ is not a multiplicative lattice since it does not satisfy the condition

$$
\langle p, q\rangle\left\langle p^{\prime}, q^{\prime}\right\rangle \leqq\langle p, q\rangle \cap\left\langle p^{\prime}, q^{\prime}\right\rangle .
$$

The original lattice, $L$, can be embedded in the lattice $L^{*}$ as follows: Let $\bar{L}$ be the sublattice of $L^{*}$ consisting of all elements of the form $\langle p, e\rangle$, where $p \in L$ and $e$ is the largest element of $L$. Then $\bar{L}$ is a residuated multiplicative lattice. In fact,

$$
\langle p, e\rangle:\langle q, e\rangle=\langle p: q, e\rangle .
$$

The mapping $\phi: L \rightarrow \bar{L}$ defined by $\phi(p)=\langle p, e\rangle$ for all $p$ in $L$ is then a lattice isomorphism of the residuated multiplicative lattice $L$ onto the residuated multiplicative lattice $\bar{L}$.

Proposition 1.2. $\bar{L}=\left\{\langle p, q\rangle \in L^{*} \mid\langle p, q\rangle \leqq\langle e, e\rangle\right\}$. If $\langle p, q\rangle \in \bar{L}$, then $\langle p, q\rangle=\langle p: q, e\rangle$.

Proof. Clearly $\langle p, e\rangle \leqq\langle e, e\rangle$ for all $p$ in $L$. If $\langle p, q\rangle \leqq\langle e, e\rangle$, then $p \leqq q$. Therefore, since $q$ is principal, $(p: q) q=p \cap q=p$. Thus, $\langle p, q\rangle=\langle q(p: q), q\rangle=\langle p: q, e\rangle$.

Let $a \in L$ and suppose that $\left\{a_{i} \mid i \in I\right\}$ is a subset of $L$. Then $a\left(\cup_{i \in I} a_{l}\right)=\cup_{t \in I} a a_{l}$. This result can be found in [6].

THEOREM 1.2. Let $p^{\prime} \in L$ such that there exists a principal nonzero divisor $A \in L$ with $a \leqq p^{\prime}$. If $q^{\prime}$ is any principal nonzero divisor in $L$, the residual $\langle p, q\rangle:\left\langle p^{\prime}, q^{\prime}\right\rangle$ exists for all elements $\langle p, q\rangle$ in $L^{*}$.

Proof. For an arbitrary element $\langle p, q\rangle \in L^{*}$, define

$$
A=\left\{\langle r, s\rangle \mid\langle r, s\rangle \in L^{*} \quad \text { and } \quad\langle r, s\rangle\left\langle p^{\prime}, q^{\prime}\right\rangle \leqq\langle p, q\rangle\right\} .
$$

$A$ is nonempty since $\langle 0, e\rangle \in A$. We will show that there exists a greatest element, $\langle c, d\rangle$, in the set $A$. It is clear that if such an element exists then $\langle c, d\rangle=\langle p, q\rangle:\left\langle p^{\prime}, q^{\prime}\right\rangle$.

We first show there exists a principal nonzero divisor $d$ in $L$ such that

(i) $\langle d, e\rangle\langle r, s\rangle \leqq\langle e, e\rangle$ for all $\langle r, s\rangle \in A$.

Let $a$ be a principal nonzero divisor such that $a \leqq p^{\prime}$. Then $\langle a, e\rangle \leqq$ $\left\langle p^{\prime}, q^{\prime}\right\rangle$ since $a q^{\prime} \leqq p^{\prime} q^{\prime} \leqq p^{\prime}$. Therefore,

$$
\langle r, s\rangle\langle a, e\rangle \leqq\langle r, s\rangle\left\langle p^{\prime}, q^{\prime}\right\rangle \leqq\langle p, q\rangle
$$


for all $\langle r, s\rangle \in A$. Hence

$$
\begin{aligned}
\langle a q, e\rangle\langle r, s\rangle & =\langle a, e\rangle\langle q, e\rangle\langle r, s\rangle \leqq\langle q, e\rangle\langle p, q\rangle \\
& =\langle q p, q\rangle=\langle p, e\rangle \leqq\langle e, e\rangle .
\end{aligned}
$$

Therefore, if we set $d=a q$, (i) is satisfied.

With $d$ defined as in the preceding paragraph, let $c=$ $\cup\{d r: s \mid\langle r, s\rangle \in A\}$. This element exists since $L$ is a complete lattice. With $c$ and $d$ defined as above, $\langle c, d\rangle$ is the greatest element of $A$. To show this, let $\langle r, s\rangle \in A$. Then $r p^{\prime} q \leqq s q^{\prime} p$. Since $\langle d r, s\rangle \leqq$ $\langle e, e\rangle, d r \leqq s$. Combining this with the fact that $s$ is principal gives

$$
(d r: s) p^{\prime} q s=(d r \cap s) p^{\prime} q=d r p^{\prime} q \leqq d q^{\prime} p s
$$

for all $\langle r, s\rangle \in A$. Therefore,

$$
(d r: s) p^{\prime} q=\left[(d r: s) p^{\prime} q s\right]: s \leqq\left(d q^{\prime} p s\right): s=d q^{\prime} p
$$

for all $\langle r, s\rangle \in A$. Thus,

$$
\bigcup_{\langle r,\rangle \in A}\left((d r: s) p^{\prime} q\right) \leqq d q^{\prime} p
$$

and so

$$
c p^{\prime} q=\left(\bigcup_{\langle r . s) \in A} d r: s\right) p^{\prime} q=\bigcup_{\langle r . s) \in A}\left((d r: s) p^{\prime} q\right) \leqq d q^{\prime} p
$$

Therefore, $\langle c, d\rangle\left\langle p^{\prime}, q^{\prime}\right\rangle \leqq\langle p, q\rangle$ and $\langle c, d\rangle$ is an element of $A$. If $\langle r, s\rangle$ is an arbitrary element of $A$ then, since $d r: s \leqq c$,

$$
r d=s(r d: s) \leqq s c
$$

Thus, $\langle r, s\rangle \leqq\langle c, d\rangle$ so $\langle c, d\rangle$ is the greatest element of $A$.

We now investigate the existence of a multiplicative inverse for elements of the lattice of fractional elements. If $\langle p, q\rangle$ is an invertible element of $L^{*},\langle p, q\rangle^{-1}$ will denote the multiplicative inverse of $\langle p, q\rangle$ in $L^{*}$. This inverse is unique if it exists.

Proposition 1.3. A nonzero element $p \in L$ is invertible in $L^{*}$ if and only if there exists an element $q \in L$ such that $p q$ is a principal nonzero divisor. 
Proof. If $\langle p, e\rangle\langle x, y\rangle=\langle e, e\rangle$, then $\langle p x, y\rangle=\langle e, e\rangle$, i.e., $p x=y$ with $y$ principal. If there exists $q \in L$ such that $p q=y$ is a principal nonzero divisor, then $\langle q, y\rangle$ is the inverse of $\langle p, e\rangle$ in $L^{*}$.

COROllary. Every principal nonzero divisor in $L$ is invertible in $L^{*}$.

Proposition 1.4. Let $\langle p, q\rangle \in L^{*}$ with $p$ a nonzero divisor. If $\langle p, q\rangle$ is invertible in $L^{*}$, then $\langle p, q\rangle^{-1}=\langle e, e\rangle:\langle p, q\rangle$.

Proof. Since $\langle p, q\rangle$ is invertible, there exists $\langle x, y\rangle \in L^{*}$ such that $p x=q y$. Thus, $p x$ is a principal nonzero divisor and $p x \leqq$ $p$. Therefore, by Theorem 1.2, $\langle e, e\rangle:\langle p, q\rangle$ exists.

Clearly, $\langle p, q\rangle^{-1} \leqq\langle e, e\rangle:\langle p, q\rangle$. Moreover,

$$
[\langle e, e\rangle:\langle p, q\rangle]\langle p, q\rangle \leqq\langle e, e\rangle
$$

Therefore,

$$
[\langle e, e\rangle:\langle p, q\rangle]\langle p, q\rangle\langle p, q\rangle^{-1} \leqq\langle p, q\rangle^{-1}\langle e, e\rangle=\langle p, q\rangle^{-1}
$$

Thus, $\langle e, e\rangle:\langle p, q\rangle \leqq\langle p, q\rangle^{-1}$.

The multiplicative lattice, $L$, is an $M$-lattice if and only if it satisfies the following condition:

(M) If $a$ and $b$ are elements of $L$ with $a \leqq b$, there exists an element $c \in L$ such that $a=b c$.

We list here two important properties of such lattices:

(1) $L$ is an $M$-lattice if and only if every element of $L$ is meet principal.

(2) An $M$-lattice is distributive.

For proofs of these properties as well as a more complete discussion of $M$-lattices, see [3] and [7].

PROPOSITION 1.5. If the nonzero elements of $L^{*}$ form a group then $L$ is an M-lattice.

Proof. Let $a$ and $b$ be elements of $L$ with $a \leqq b$. Then there exists $\langle x, y\rangle \in L^{*}$ such that

(i) $\langle b, e\rangle\langle x, y\rangle=\langle a, e\rangle$.

Thus, $b x=a y$ with $y$ a principal nonzero divisor in $L$. Since $a \leqq b$, $a=a \cap b$ and so

$$
b x=a y=(a \cap b) y=a y \cap b y=b x \cap b y .
$$


Thus, $b x \leqq b y$ and therefore $x \leqq y$. Thus, by Proposition $1.2,\langle x, y\rangle=$ $\langle x: y, e\rangle$. Therefore, (i) may be rewritten

$$
\langle b, e\rangle\langle x: y, e\rangle=\langle a, e\rangle
$$

or, $b(x: y)=a$.

THEOREM 1.3. The nonzero elements of $L^{*}$ from a group if and only if every nonzero element of $L$ is a principal nonzero divisor.

Proof. If the nonzero elements of $L^{*}$ from a group then $L$ is an $M$-lattice by the previous proposition so that every element of $L$ is meet principal. To show every element is join principal, let $a, b \in L$, $b \neq 0$. Then $(a b: b) b \leqq a b$ which implies $a b: b \leqq a$ since $b$ has an inverse in $L^{*}$. Since clearly $a \leqq a b: b$, we have

(i) $a b: b=a$

for all $a, b \in L, b \neq 0$.

Let $a, b, c$ be elements of $L$ with $c \neq 0$. Then

$$
((a: c) \cup b) c=(a: c) c \cup b c=(a \cap c) \cup b c
$$

since $c$ is meet principal. Since $L$ is distributive,

$$
(a \cap c) \cup b c=(a \cup b c) \cap(c \cup b c)=(a \cup b c) \cap c .
$$

Thus,

(ii) $((a: c) \cup b) c=(a \cup b c) \cap c$.

Using equations (i) and (ii) gives

$$
\begin{aligned}
(a: c) \cup b & =[((a: c) \cup b) c]: c=[(a \cup b c) \cap c]: c \\
& =(a \cup b c): c .
\end{aligned}
$$

Thus, every nonzero element of $L$ is a principal nonzero divisor.

Conversely, if every nonzero element of $L$ is a principal nonzero divisor and if $\langle p, q\rangle \in L^{*},\langle p, q\rangle \neq\langle 0, e\rangle$, then $\langle q, p\rangle \in L^{*}$. Thus

$$
\langle p, q\rangle\langle q, p\rangle=\langle e, e\rangle
$$

so $\langle p, q\rangle$ is invertible in $L^{*}$.

Proposition 1.6. If every nonzero element of $L$ is invertible in $L^{*}$ then the nonzero elements of $L^{*}$ from a group. 
Proof. Let $\langle p, q\rangle \in L^{*}, p \neq 0$. Since $p$ is invertible in $L^{*}$, there exists $\langle x, y\rangle \in L^{*}$ such that $p x=y$. Then $\langle x q, y\rangle$ is the multiplicative inverse for $\langle p, q\rangle$ in $L^{*}$.

Proposition 1.7. Suppose L satisfies the following conditions:

(1) Every element of $L$ contains a principal element.

(2) L contains no zero divisors.

Then $L$ is an M-lattice if and only if every nonzero element of $L$ is a principal nonzero divisor.

Proof. If every element is principal, $L$ is clearly an $M$ lattice. Suppose $L$ is an $M$-lattice and let $p \in L, p \neq 0$. Let $q \leqq p$ be a principal element of $L$. Then $q=p r$ for some $r$ in $L$. Thus, $p$ is invertible in $L^{*}$ by Proposition 1.3. The Proposition then follows from Proposition 1.6 and Theorem 1.3.

ExAmple. Let $L(R)$ be the lattice of ideals of a commutative ring with identity $R$. Let $L(Q(R))$ denote the lattice of fractional ideals of $R$. If $A \in L(Q(R))$, then $A=\frac{1}{d} B$, where $B$ is an ideal of $R$. The mapping $\phi: L(Q(R)) \rightarrow L^{*}$ defined by $\phi\left(\frac{1}{d} B\right)=\langle B,(d)\rangle$ is an isomorphism of $L(Q(R))$ onto $L^{*}$. Thus, in this case, the lattice of fractional elements defined above is isomorphic to the lattice of fractional ideals of $R$.

2. Dedekind lattices. Throughout this section we will assume that $L$ is a multiplicative lattice that satisfies the following conditions:

(A) $L$ is modular.

(B) Every element of $L$ is a join of principal elements.

(C) If $p$ is a principal element of $L$ and $p \leqq U_{t \in I} q_{t}$ where each $q_{t}$ is principal, then there exists a finite subset $I^{\prime}$ of $I$ such that $p \leqq \cup_{i \in I^{\prime}} q_{i}$.

(D) $L$ contains no zero divisors.

$L^{*}$ will denote the lattice of fractional elements of $L$.

If $L(R)$ is the lattice of ideals of a commutative ring with identity $R$, then $L(R)$ satisfies (A) and (B). Since every principal element of $L(R)$ is a finitely generated ideal of $R([3]$, p. 655), $L(R)$ also satisfies (C). We also remark that a Noether lattice satisfies (A) through (C). A further discussion of (B) and (C) can be found in [6].

Definition 2.1. A Dedekind lattice is a multiplicative lattice satisfying (A) through (D) above in which every element can be written 
as a finite product of prime elements.

Lemma 2.1. Let $\left\{p_{i} \mid i=1, \cdots, n\right\}$ be a set of elements of $L$. If $\prod_{i=1}^{n} p_{i}$ is invertible in $L^{*}$, then each $p_{i}$ is invertible in $L^{*}$.

Proof. By Proposition 1.3, $\prod_{i=1}^{n} p_{i}$ is invertible if and only if there exists $x, y \in L$ with $y$ principal such that $x \prod_{i=1}^{n} p_{i}=y$. Then, for $\mathrm{\jmath}=1, \cdots, n$,

$$
p_{i}\left(x \prod_{i \neq j} p_{i}\right)=y
$$

so $p_{j}$ is invertible by Proposition 1.3.

Lemma 2.2. For products of invertible prime elements of $L$, the factorization into prime elements is unique.

Proof. Suppose $a=\prod_{i=1}^{n} p_{i}=\prod_{j=1}^{m} q_{j}$ where $p_{i}$ and $q_{j}$ are prime in $L$ and $a$ is invertible in $L^{*}$. Further, assume $p_{1}$ is minimal among the set $\left\{p_{i} \mid i=1, \cdots, n\right\}$. Then $\Pi_{j=1}^{m} q_{j} \leqq p_{1}$ so there exists $q_{j}$ such that $q_{j} \leqq$ $p_{1}$. Without loss of generality we may assume $j=1$ so that $q_{1} \leqq$ $p_{1}$. Now, $\Pi_{i=1}^{n} p_{i} \leqq q_{1}$. Thus, there exists an integer $s$ such that $p_{s} \leqq q_{1}$. Then $p_{s} \leqq q_{1} \leqq p_{1}$ which implies $q_{1}=p_{1}$ since $p_{1}$ was assumed to be minimal among the $p_{i}$. By Lemma $2.1, p_{1}$ is invertible in $L^{*}$. Therefore,

$$
\prod_{i=2}^{n} p_{i}=p_{1}^{-1} p_{1} \prod_{i=2}^{n} p_{i}=p_{1}^{-1} p_{1} \prod_{j=2}^{m} q_{j}=\prod_{j=2}^{m} q_{j}
$$

Clearly, $\prod_{i=2}^{n} p_{i}=\prod_{j=2}^{m} q_{j}$ is invertible in $L^{*}$, so the above argument can be repeated.

Proposition 2.1. If $p \in L$ is invertible in $L^{*}$, then $p$ can be written as a finite join of principal elements.

Proof. If $p \in L$ is invertible in $L^{*}$ there exists $\langle r, s\rangle \in L^{*}$ such that $\langle p, e\rangle\langle r, s\rangle=\langle e, e\rangle$. By condition (B) on the lattice $L$, we can write

$$
p=\bigcup_{i \in I} p_{i} \quad \text { and } \quad r=\bigcup_{j \in J} r_{j}
$$

where $p_{i}$ and $r_{j}$ are principal for all $i \in I$ and all $j \in J$. Therefore, 


$$
\begin{aligned}
\langle e, e\rangle & =\langle p, e\rangle\langle r, s\rangle=\left\langle\bigcup_{i \in I} p_{i}, e\right\rangle\left\langle\bigcup_{j \in J} r_{j}, e\right\rangle \\
& =\left\langle\bigcup_{i, j}\left(p_{i} r_{j}\right), s\right\rangle .
\end{aligned}
$$

Thus, $s=\cup_{i, j}\left(p_{i} r_{j}\right)$. Since $s$ is principal, by condition (C), $s$ can be written as a join of finitely many of the elements $p_{i} r_{j}$. Thus,

$$
s=\bigcup_{k=1}^{n} p_{k} r_{k}
$$

where, for all $k, p_{k} \leqq p$ and $r_{k} \leqq r$ and $p_{k}, r_{k}$ are principal. Therefore $\langle e, e\rangle=\cup_{k=1}^{n}\left(\left\langle p_{k}, e\right\rangle\left\langle r_{k}, s\right\rangle\right)$ and so,

$$
\begin{aligned}
\langle p, e\rangle & =\langle p, e\rangle\langle e, e\rangle=\bigcup_{k=1}^{n}\left(\langle p, e\rangle\left\langle p_{k}, e\right\rangle\left\langle r_{k}, s\right\rangle\right) \\
& \leqq \bigcup_{k=1}^{n}\left(\langle p, e\rangle\langle r, s\rangle\left\langle p_{k}, e\right\rangle\right)=\bigcup_{k=1}^{n}\langle e, e\rangle\left\langle p_{k}, e\right\rangle \\
& =\bigcup_{k=1}^{n}\left\langle p_{k}, e\right\rangle .
\end{aligned}
$$

Since $p_{k} \leqq p$ for all $k$,

$$
p=\bigcup_{k=1}^{n} p_{k}
$$

Proposition 2.2. If $p \in L$ is invertible in $L^{*}$, then $q p: p=q$ for all $q \in L$.

Proof. Clearly $q \leqq q p: p$. Moreover, $(q p: p) p \leqq q p$ and so, since $p$ is invertible,

$$
q p: p=(q p: p) p p^{-1} \leqq q p p^{-1}=q .
$$

THEOREM 2.1. In a Dedekind lattice every proper, nonzero prime element is maximal in $L$ and invertible in $L^{*}$.

Proof. We first show that every invertible prime of $L$ is maximal. Because of condition (B) it will suffice to show that if $q \in L$ is principal and $q \not \equiv p$, then $p \cup q=e$. Thus, assume $q \in L$ is principal and $q \not \equiv$ and consider the elements $p \cup q$ and $p \cup q^{2}$. Since $L$ is a Dedekind lattice 
(i) $p \cup q=\prod_{i=1}^{r} p_{i}$
(ii) $p \cup q^{2}=\prod_{j=1}^{s} q_{i}$

where $p_{i}$ and $q_{j}$ are prime. Clearly, $p \cup q, p \cup q^{2}$ as well as the elements $p_{i}$ and $q_{j}$ belong to the factor lattice $L / p$. We will denote elements of $L / p$ by $a / p, b / p$, etc.

Since $p$ is prime in $L, L / p$ has no zero divisors and since $q$ and $q^{2}$ are principal in $L,(p \cup q) / p$ and $\left(p \cup q^{2}\right) / p$ are principal in $L / p$.

Let $(L / p)^{*}$ denote the lattice of fractional elements of $L / p$. Since $(p \cup q) / p$ and $\left(p \cup q^{2}\right) / p$ are principal nonzero divisors in $L / p$, they are invertible in $(L / p)^{*}$ by the Corollary to Proposition 1.3. The elements $p_{i} / p$ and $q_{j} / p$ are prime in $L / p$ since they are prime in $L$. Thus (i) and (ii) give $(p \cup q) / p$ and $\left(p \cup q^{2}\right) / p$ as a product of primes of $L / p$.

Since $\Pi_{i=1}^{r}\left(p_{i} / p\right)=(p \cup q) / p$ is invertible in $(L / p)^{*}$, each $p_{i} / p$ is invertible in $(L / p)^{*}$ by Lemma 2.1. Similarly, each $q_{j} / p$ is invertible in $(L / p)^{*}$.

We now note that $p \cup(p \cup q)^{2}=p \cup q^{2}$. Therefore, in $L / p$

$$
\prod_{i=1}^{r}\left(p_{i} / p\right)^{2}=(p \cup q)^{2} / p=\left(p \cup q^{2}\right) / p=\prod_{j=1}^{s}\left(q_{j} / p\right) .
$$

Thus, since each $p_{i} / p$ and $q_{j} / p$ is invertible in $(L / p)^{*}$, by Lemma 2.2 the $q_{j} / p$ must be the $p_{i} / p$ each repeated twice. Specifically, in $L / p$ we have $s=2 r$ and after a possible renumbering of the $q_{j}, q_{2 i} / p=q_{2 i-1} / p=$ $p_{i} / p$. Therefore, since $p_{i} \geqq p$ for all $i$ and $q_{j} \geqq p$ for all $j$,

$$
q_{2 i}=q_{2 i-1}=p_{i}
$$

in the lattice $L$. Therefore, in the lattice $L$,

$$
\text { (iii) } \begin{aligned}
p & \leqq p \cup q^{2}=\prod_{j=1}^{s} q_{j}=\prod_{i=1}^{r} p_{i}^{2}=(p \cup q)^{2}=p^{2} \cup q(p \cup q) \\
& \leqq p^{2} \cup q .
\end{aligned}
$$

Since $p$ is prime and $q \not p, r q \leqq p$ implies that $r \leqq p$. Therefore, $p: q \leqq p$ and so $p \cap q=(p: q) q \leqq p q$, where the first equality follows from the fact that $q$ is principal. Since $L$ is a multiplicative lattice, $p q \leqq p \cap q$ and therefore

(iv) $p q=p \cap q$ (iv),

By assumption, $p$ is invertible. Therefore, by Proposition 2.2 and

(v) $q=q p: p=(q \cap p): p=q: p$.

We now establish the following equation: 
(vi) $\left(p^{2} \cup q\right): p=p^{2}: p \cup q: p$.

By Proposition 2.2, $\left(p^{2}: p\right) p=p^{2}$ and by (iv) and (v), $(q: p) p=$ $q p=q \cap p$. Therefore

$$
\begin{aligned}
\left(p^{2}: p\right) \cup(q: p) & =\left(\left(p^{2}: p\right) \cup(q: p)\right) p: p=\left(\left(p^{2}: p\right) p \cup(q: p) p\right): p \\
& =\left(p^{2} \cup(p \cap q)\right): p=\left(\left(p^{2} \cup q\right) \cap p\right): p=\left(p^{2} \cup q\right): p
\end{aligned}
$$

where we have again used Proposition 2.2 as well as the fact that $L$ is modular.

By equation (iii), $p \leqq p^{2} \cup q$. Therefore, using (vi) and Proposition 2.2 gives

$$
e=p: p \leqq\left(p^{2} \cup q\right): p=\left(p^{2}: p\right) \cup(q: p)=p \cup(q: p)=p \cup q .
$$

Therefore, $p \cup q=e$ and every invertible prime is maximal. We now show that every prime is invertible. Let $p$ be prime and let $q$ be a principal element with $q \leqq p$. Then $q=\prod_{i=1}^{n} p_{i}$ where each $p_{i}$ is prime. Since $q$ is principal it is invertible in $L^{*}$. Therefore $p_{i}$ is invertible in $L^{*}$ for all $i$. Thus, each $p_{i}$ is maximal in $L$ by the first part of the proof. But this implies that $p_{i}=p$ for some $i$ and so $p$ is invertible.

Corollary 2.1. In a Dedekind lattice, the factorization of an element into a product of primes is unique.

Corollary 2.2. In a Dedekind lattice every nonzero element is invertible in $L^{*}$.

Proof. If $a \in L, a \neq 0$, then $a=\prod_{i=1}^{n} p_{i}$ with $p_{i}$ prime for all $i$.

By Theorem 2.6 and Proposition 1.3, there exists $b_{i} \in L$ such that $p_{i} b_{i}$ is principal. Then, if $b=\prod_{i=1}^{n} b_{i}, a b$ is principal so $a$ is invertible by Proposition 1.3.

\section{Corollary 2.3. A Dedekind lattice is a Noether lattice.}

Proof. If $L$ is a Dedekind lattice then every nonzero element of $L$ is invertible by Corollary 2.2. Thus, by Proposition 2.1 every element of $L$ can be written as a finite join of principal elements. By using conditions (B) and (C) imposed on $L$ one can prove exactly as in the ring theoretic case, that $L$ then satisfies the $A C C$. Thus, $L$ is a Noether lattice.

Dilworth [1] has noted a special case of the following theorem. By using Corollary 2.3 and Theorem 1.3, his proof can be extended to the 
present case. It is also possible to give a proof using Theorem 5 of [4]. We give a different proof.

THEOREM 2.2. A multiplicative lattice, L, satisfying conditions (A) through (D) is a Dedekind lattice if and only if the nonzero elements of $L^{*}$ form a group.

Proof. If $L$ is Dedekind every element of $L$ is invertible so the nonzero elements of $L^{*}$ form a group by Proposition 1.6.

If the nonzero elements of $L^{*}$ form a group, every element of $L$ is principal by Theorem 1.3. Thus $L$ is a Noether lattice. Let $S$ be the set of elements that cannot be written as a product of prime elements. If $S$ is nonempty it contains a maximal element, $a$, since $L$ is a Noether lattice. Now, $a$ is not maximal in $L$ since every maximal element of $L$ is prime. Let $m$ be a maximal element of $L$ such that $a \leqq m$. Such an element exists since $L$ is a Noether lattice.

Consider $a: m$. Clearly $a \leqq a: m$. Moreover $a \neq a: m$. For suppose $a=a: m$. Then, since $m$ is principal and $a$ is invertible in $L^{*}$,

$$
m=a^{-1} a m=a^{-1}(a: m) m=a^{-1}(a \cap m)=a^{-1} a=e .
$$

Thus, $a<a: m$, so $a: m$ is a product of primes, that is, $a: m=p_{1} \cdots p_{n}$, where each $p_{i}$ is a prime. Then, since $m$ is principal

$$
a=a \cap m=(a: m) m=p_{1} \cdots p_{n} m
$$

is a representation of $a$ as a product of prime elements. The contradiction establishes the Theorem.

The following result is an immediate consequence of the preceding theorem, Proposition 1.7, and Theorem 1.3.

COROLlaRY 2.4. A multiplicative lattice satisfying (A) through (D) is a Dedekind lattice if and only if it is an M-lattice.

From the corollary to Theorem 6 of [3], it follows that an $M$-lattice satisfying (A) through (D) also satisfies the $A C C$. In [8], M. Ward has investigated $M$-lattices satisfying the $A C C$. By using the primary decomposition, he has shown that every element of such a lattice has a unique decomposition into a product of prime elements ([8], Theorem 5.2). This result, together with Proposition 1.5, could also be used to prove Theorem 2.2. Using Corollary 2.4, we also obtain the following restatement of Theorem 6.1 of [8]. 
and only if it is a Noether lattice without zero divisors satisfying

(i) Every primary element of $L$ is a power of a prime;

(ii) If $p$ is prime, $p \leqq q$, and $p \neq q$, then $q p=p$.

For the following theorem, we use the definition of integrally closed elements given in [5].

THEOREM 2.4. A multiplicative lattice satisfying (A) through (D) is a Dedekind lattice if and only if $L$ satisfies the following conditions :

(1) L is a Noether lattice;

(2) Every nonzero prime element of $L$ is maximal;

(3) Every principal element of $L$ is integrally closed.

Proof. Assume $L$ satisfies (1) through (3) and let $p$ be a prime in $L$. Let $a \leqq p$ be a principal element. By (2) $p$ is a minimal prime associated with $a$.

In [2], Furuyama has defined the $n$th symbolic primary power $q^{(n)}$ of a primary element $q$ associated with $p$ to be $\left(q^{n}\right)_{p}$, where $\left(q^{n}\right)_{p}$ denotes the $p$-primary component of $q^{n}$. He has then shown that if $p$ is a prime associated with a principal integrally closed element, the only $p$-primary elements are the symbolic powers $p^{(n)}$. Thus, the symbolic powers $p^{(n)}$ are the only $p$-primary elements of $L$. Therefore, the quotient lattice $L_{p}$ is totally ordered, the only elements of $L_{p}$ being the powers $[p]^{n}$ of the maximal element $[p]$. By Theorem 6 of [6], this implies that $L$ is an $M$-lattice. Thus, by Proposition 1.7 and Theorems 1.2 and $2.2, L$ is a Dedekind lattice.

Conversely, suppose $L$ is Dedekind. By Corollary $2.3, L$ is a Noether lattice and by Theorem 2.1, every prime is maximal. Suppose $a$ is $a$-dependent on $b$ (for a definition of this relation, see [5]). Then there exists an integer $n$ such that $(a \cup b)^{n+1}=b(a \cup b)^{n}$. Since $L$ is Dedekind, every element of $L$ is invertible in $L^{*}$. Thus,

$$
a \cup b=(a \cup b)^{n+1}(a \cup b)^{-n}=b(a \cup b)^{n}(a \cup b)^{-n}=b .
$$

Therefore, $a \leqq b$, so $b$ is integrally closed.

Since, by Corollary 2.3, a Dedekind lattice is a Noether lattice, the following theorem is an obvious consequence of Theorem 5 of [4].

TheOREM 2.5. A Dedekind lattice is isomorphic to the lattice of ideals of a Noetherian ring.

The following result follows from the corresponding ring theoretic result by using Theorem 2.5. A lattice theoretic proof can also be given 
which is exactly analogous to the ring theoretic proof.

Corollary 2.5. Let $L$ be a Dedekind lattice. Then every element $\langle a, b\rangle$ of $L^{*}$ can be written uniquely in the form

$$
\langle a, b\rangle=\prod_{\substack{p \in L \\ p \text { prime }}} p^{n_{p}((a, b))}
$$

where $n_{p}(\langle a, b\rangle)$ is an integer and $n_{p}(\langle a, b\rangle)=0$ for all but finitely many $p$ in $L$. The following equations also hold:

(1) $n_{p}(\langle a, b\rangle \cup\langle c, d\rangle)=\min \left\{n_{p}(\langle a, b\rangle), n_{p}(\langle c, d\rangle)\right\}$

(2) $n_{p}(\langle a, b\rangle \cap\langle c, d\rangle)=\max \left\{n_{p}(\langle a, b\rangle), n_{p}(\langle c, d\rangle)\right\}$

(3) $n_{p}(\langle a, b\rangle\langle c, d\rangle)=n_{p}(\langle a, b\rangle)+n_{p}(\langle c, d\rangle)$

(4) $\langle a, b\rangle \leqq\langle c, d\rangle$ iff $n_{p}(\langle a, b\rangle) \geqq n_{p}(\langle c, d\rangle)$ for all primes $p$ in $L$.

\section{REFERENCES}

1. R. P. Dilworth, Abstract commutative ideal theory, Pacific J. Math., 12 (1962), 481-498.

2. E. I. Furuyama, Unpublished Ph.D. dissertation, Washington State University, 1972.

3. M. F. Janowitz, Principal multiplicative lattices, Pacific J. Math., 33 (1970), 653-656.

4. E. W. Johnson and J. P. Lediaev, Representable distributive Noether lattices, Pacific J. Math., 28 (1969), 561-564.

5. John P. Lediaev, Asymptotic and integral closure of elements in multiplicative lattices, Michigan Math. J., 16 (1968), 235-243.

6. P. J. McCarthy, Arithmetical rings and multiplicative lattices, Ann. Mat. Pura Appl., 82 (1969), 267-274.

7. P. J. McCarthy, Note on abstract commutative ideal theory, Amer. Math. Monthly, 74 (1967), 706-707.

8. M. Ward, Residuated distributive lattices, Duke Math. J., 6 (1940), 641-651.

9. O. Zariski and P. Samuel, Commutative Algebra I, Princeton: Van Nostrand, 1960.

Received October 26, 1973. This research constitutes a portion of a doctoral dissertation written under the direction of Professor William M. Cunnea at Washington State University.

WASHINGTON STATE UnIVERSITY 




\section{Pacific Journal of Mathematics}

\section{Vol. 56, No. $1 \quad$ November, 1975}

Shimshon A. Amitsur, Central embeddings in semi-simple rings .......... 1

David Marion Arnold and Charles Estep Murley, Abelian groups, A, such

that $\operatorname{Hom}(A,---)$ preserves direct sums of copies of $A \ldots \ldots \ldots .$.

Martin Bartelt, An integral representation for strictly continuous linear

operators ................................... 21

Richard G. Burton, Fractional elements in multiplicative lattices......... 35

James Alan Cochran, Growth estimates for the singular values of

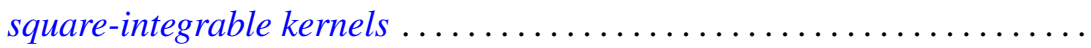

C. Martin Edwards and Peter John Stacey, On group algebras of central

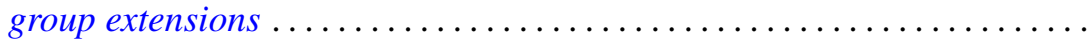

Peter Fletcher and Pei Liu, Topologies compatible with homeomorphism

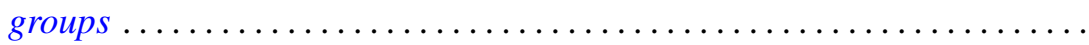

George Gasper, Jr., Products of terminating ${ }_{3} F_{2}(1)$ series ............ 87

Leon Gerber, The orthocentric simplex as an extreme simplex ............

Burrell Washington Helton, A product integral solution of a Riccati

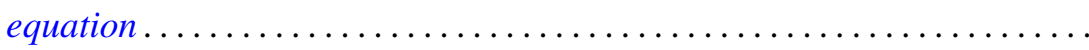

Melvyn W. Jeter, On the extremal elements of the convex cone of

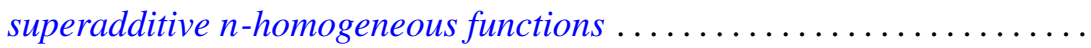

R. H. Johnson, Simple separable graphs .

Margaret Humm Kleinfeld, More on a generalization of commutative and

alternative rings. . .

A. Y. W. Lau, The boundary of a semilattice on an $n$-cell.

Robert F. Lax, The local rigidity of the moduli scheme for curves ...

Glenn Richard Luecke, A note on quasidiagonal and quasitriangular

operators .

Paul Milnes, On the extension of continuous and almost periodic functions

Hidegoro Nakano and Kazumi Nakano, Connector theory.

James Michael Osterburg, Completely outer Galois theory of perfect rings ..................................

Lavon Barry Page, Compact Hankel operators and the F. and M. Riesz

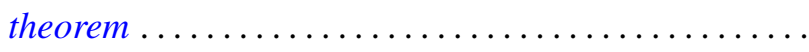

Joseph E. Quinn, Intermediate Riesz spaces..................... 225

Shlomo Vinner, Model-completeness in a first order language with a generalized quantifier.

Jorge Viola-Prioli, On absolutely torsion-free rings ..........

Philip William Walker, A note on differential equations with all solutions of integrable-square............................

Stephen Jeffrey Willson, Equivariant maps between representation 